\title{
Medo de quedas em idosos: uma revisão da literatura
}

\author{
Fear of falls in the elderly: a literature review
}

\author{
Miedo a caídas en adultos mayores: una revisión de la literatura
}

\author{
Flávia M. Malini*, Cláudia S. Lopes, Roberto A. Lourenço
}

\begin{abstract}
Resumo
O medo de quedas vem ganhando reconhecimento como um importante problema de saúde nos idosos, inclusive naqueles sem histórico de queda anterior. Porém, no Brasil, tanto na prática clínica como na de pesquisa, o medo de quedas é pouco avaliado. Isso talvez seja devido à falta de instrumentos estruturados adaptados e que possam ser utilizados com nossa população de idosos. Poucos estudos foram realizados no Brasil com a finalidade de se conhecer a prevalência e características do medo de quedas. O objetivo deste artigo é revisar a literatura sobre a prevalência e os fatores associados ao medo de quedas, contribuindo para este importante problema de saúde. O medo de quedas tem alta prevalência nessa população, o que interfere diretamente na qualidade de vida do indivíduo, sendo relacionado a desfechos desfavoráveis de saúde, tais como restrição de atividades, depressão, isolamento social e novas quedas. Portanto, identificar os fatores envolvidos no medo de quedas é de extrema importância para o rastreio, identificação e acompanhamento de idosos com risco de declínio funcional e que necessitam de intervenções com o objetivo de manter por maior tempo possível sua capacidade funcional, independência e autonomia.
\end{abstract}

Descritores: Idoso; Acidentes por quedas; Medo.

\begin{abstract}
The fear of falling has been gaining recognition as an important health problem in the elderly, even in those without a history of previous fall. However, in Brazil, both in clinical practice and in the research field, fear of falling has been scarcely assessed. This might be due to a lack of structured adapted instruments that can be used on our elderly population. Few studies have been conducted in Brazil in order to determine the prevalence and the characteristics of the fear of falling. The purpose of this article is to review the literature regarding the prevalence and associated factors of fear of falling, contributing to the knowledge about this important health problem. Fear of falling is highly prevalent in this population, and it can directly affect the quality of life of the individuals, being related to adverse health outcomes, such as activity restriction, depression, social isolation and further falls. Thus, identifying the factors involved in the fear of falling is of extreme importance for screening, identification and monitoring of elderly patients in risk of functional decline who need interventions in order to maintain for as long as possible their functional capacity, independence and autonomy.
\end{abstract}

Keywords: Aged; Accidental falls; Fear.

\section{Resumen}

El miedo a caídas viene ganando reconocimiento como un problema importante de salud en los adultos mayores, incluso en aquellos sin antecedentes de caída anterior. Sin embargo, en Brasil, tanto en la práctica clínica como en la investigación, el miedo a caer es poco evaluado. Tal vez eso se deba a la falta de instrumentos estructurados, adaptados, que puedan ser utilizados en nuestra población de adultos mayores. Pocos estudios fueron realizados en Brasil con la finalidad de conocerse la prevalencia y características del miedo a caídas. El objetivo de este artículo es revisar la literatura sobre la prevalencia y los factores asociados al miedo a caídas, contribuyendo con este importante problema de salud. El miedo a caídas tiene alta prevalencia en esta población, lo que interfiere 
directamente en la calidad de vida del individuo, siendo relacionado a desenlaces desfavorables de salud, tales como la restricción de actividades, la depresión, el aislamiento social y las nuevas caídas. Por lo tanto, identificar los factores desarrollados en el miedo a caídas es de extrema importancia para el rastreo, identificación y acompañamiento de los adultos mayores con riesgo potencial de declive funcional, que necesitan de intervenciones con el objetivo de mantener por el mayor tiempo posible su capacidad funcional, su independencia y autonomía.

Palabras clave: Anciano; Accidentes por caídas; Miedo.

\section{Introdução}

O medo de quedas ou ptofobia foi descrito inicialmente por Murphy e colaboradores, em 1982, como parte da síndrome pós-queda. Acreditava-se que, após sofrer uma queda, além das consequências físicas diretas desta, os idosos também apresentariam consequências psicológicas, como medo de uma nova queda e perda de autoestima. O medo foi identificado como sendo o componente central desta síndrome.' Porém, estudos mais recentes demonstram: mesmo os idosos que não vivenciaram uma queda podem apresentar o medo de cair. ${ }^{2,3}$

A relação entre o medo de quedas e as quedas parece ser bidirecional: o medo parece ser mais frequente entre indivíduos que caíram, e pessoas com esse medo estão mais propensas a cair. ${ }^{4}$

As quedas e o medo de quedas são condições que podem gerar desfechos graves à saúde dos idosos e possuem fatores de risco compartilhados, podendo levar os indivíduos com tais condições a um ciclo vicioso de declínio funcional, isolamento social, descondicionamento físico, alteração de equilíbrio e marcha, perda da confiança, entre outros, aumentando o risco de quedas e o medo destas. $^{4}$

$\mathrm{O}$ medo de quedas foi definido por Tinetti e Powell ${ }^{5}$ como uma preocupação constante com a possibilidade de cair que limita o desempenho das atividades de vida diária. Porém, muitas definições são encontradas na literatura, como a habilidade do indivíduo para controlar o ambiente, sua mobilidade e a habilidade de prevenir ou manejar quedas; consequências negativas relacionadas ao medo, como restrição de atividades e diminuição de qualidade de vida; e até pensamentos do indivíduo sobre o manejo de uma eventual queda, como por exemplo, formas de se levantar do chão e como se proteger das quedas. ${ }^{6,7}$

Atualmente, a definição mais utilizada internacionalmente é fundamentada no conceito de autoeficácia. De acordo com a teoria cognitiva social (TCS) proposta por Bandura ${ }^{8}$, o indivíduo, em face de circunstâncias desafiadoras, deve ter não apenas as habilidades necessárias, mas também a crença de que é capaz de lidar com elas. Portanto, a autoeficácia é uma combinação de habilidades, motivação e confiança. ${ }^{8,9}$ Essa teoria, quando aplicada ao medo de quedas, representa o grau de confiança que a pessoa tem para realizar as atividades do dia a dia sem cair. ${ }^{5}$

A autoeficácia é influenciada não apenas por habilidades e estado físico, mas também pelas experiências prévias, pela observação da experiência de outros e de suas consequências, e sofre influência social. Desta forma, ela é tanto influenciada por desempenhos passados como preditora de desempenhos futuros. ${ }^{10}$

De acordo com diversos estudos, a forma mais utilizada para mensurar o medo de quedas é através do conceito de autoeficácia, a qual possui maior reconhecimento científico por ser fundamentada na TCS. Sendo assim, para muitos autores, a autoeficácia constitui o principal elemento do medo de quedas, sendo considerada como o componente cognitivo do medo. Os outros componentes seriam o fisiológico, como por exemplo um aumento nas respostas de reatividade dos sistemas, e o componente comportamental, que seria, por exemplo, uma diminuição da velocidade de marcha. ${ }^{11,12}$

Desde que Tinetti e colaboradores ${ }^{13}$ contextualizaram o medo de quedas como uma baixa autoeficácia - já que, aplicado a esse contexto, ele implica em pensamentos sobre quedas e, na falta de confiança na própria capacidade de evitá-las -, esta vem sendo considerada a estratégia válida de avaliação do medo de quedas.

Utilizado também como um domínio para avaliação da funcionalidade, o conceito de autoeficácia vem sendo reconhecido como componente essencial de desempenho e bem-estar nos idosos, pois está associado a envelhecimento, incapacidades e doenças. ${ }^{14}$ 
A importância de se avaliar e prevenir o medo de quedas fundamenta-se nos achados que mostram que, depois que o medo se desenvolveu, é altamente provável que ele persista independente de haver uma queda ou não, mostrando o quanto esta condição pode interferir na vida dos indivíduos. ${ }^{4}$

Apesar de existirem circunstâncias nas quais o medo de queda pode ser considerado um fator protetor, quando, por exemplo, este impede que o indivíduo se arrisque em situações que poderiam provocar quedas, em outras, este medo pode ser exagerado ou desproporcional em relação ao estímulo. Esse medo então leva a consequências deletérias à saúde dos idosos, interferindo na qualidade de vida, no conforto emocional e no desempenho do indivíduo, restringindo sua participação social e provocando isolamento..$^{12,15}$

É preciso identificar os indivíduos que necessitam de intervenção, direcionar quais atividades devem ser focadas no planejamento do tratamento e verificar a efetividade das intervenções oferecidas. As consequências relacionadas ao medo de quedas podem ser tão ou mais incapacitantes do que a própria queda e são eventos comuns e potencialmente preveníveis na população idosa. ${ }^{16}$

\section{Prevalência e incidência do medo de quedas}

Devido às diferentes formas utilizadas para mensurar o medo de quedas, à falta de uniformidade entre elas, à utilização de populações distintas e a diferenças metodológicas nas pesquisas, a prevalência do medo de quedas é variável entre os estudos, podendo assumir valores entre $12 \%$ e 65\% em idosos independentes e sem histórico de quedas, e entre $29 \%$ e $92 \%$ naqueles com história de quedas. ${ }^{3}$

Alguns estudos utilizam apenas uma pergunta simples e direta sobre a presença do medo de quedas - "Você tem medo de quedas?" -, com duas opções de resposta: sim ou não. Essa é uma maneira rápida de gerar estimativas de prevalência, que podem ser úteis para o rastreio, porém não conseguem captar as variações de intensidade do medo. ${ }^{17,18}$

Baixas prevalências foram encontradas em estudos que utilizam essa pergunta, o que levou ao estabelecimento de perguntas com mais opções de resposta, buscando captar diferentes intensidades do medo. ${ }^{19,20}$

Nos últimos anos, alguns instrumentos foram desenvolvidas para mensurar o medo de quedas. São exemplos de tais instrumentos: Falls Efficacy Scale (FES), Falls Efficacy Scale-International (FES-I), Activities Specific Balance Confidence Scale (ABC), Survey of Activities and Fear of Falling in the Elderly (SAFE), Perceived Control Over Falling e Perceived Ability to Manage Falls and Falling. . $^{3,15}$

A Falls Efficacy Scale International (FES-I $-B R)^{21}$ é a única escala que foi estudada na língua portuguesa, por Camargos e colaboradores, ${ }^{21} \mathrm{em}$ 2010, e por esta razão seus resultados são passíveis de comparação com estudos internacionais.

$\mathrm{Na}$ literatura brasileira, poucos trabalhos analisaram a prevalência do medo de quedas. No estudo realizado por Lopes e colaboradores, ${ }^{22}$ a presença de medo foi avaliada por meio da FES-I -BR, em uma amostra de 147 idosos do Programa de Saúde da Família, no qual 90,48\% dos idosos apresentaram medo em, no mínimo, uma das 16 atividades propostas pela escala. Dentre essas atividades, as que apresentaram maior relato de preocupação foram: andar em superfície escorregadia, caminhar sobre uma superfície irregular, subir e descer escadas, subir e descer ladeira e tomar banho.

No estudo realizado por Dias e colaboradores, ${ }^{23}$ o medo de quedas foi avaliado por meio de um item com quatro categorias de resposta: "não", "um pouco", "moderado" e "muito". Neste estudo, também foi avaliado o conceito de autoeficácia, pela FES-I-BR, e o de restrição de atividades devido ao medo. Este estudo avaliou 113 idosos comunitários e encontrou uma prevalência de $28,32 \%$ de medo de queda através da pergunta; desses que relataram medo, 52,22\% disseram ter reduzido suas atividades devido a esse medo.

Em uma revisão sistemática realizada por Scheffer e colaboradore ${ }^{12}$ em 2008, foram identificados apenas dois estudos sobre a incidência do medo, pois os idosos investigados eram indivíduos sem quedas que, durante o seguimento, desenvolveram o medo. Nesses estudos, a incidência variou entre 11,6 e $23,3 \%$ em idosos sem quedas durante o seguimento, e entre 20,6 e $39 \%$ em idosos que desenvolveram o medo após uma queda. 
Em estudo de seguimento realizado por dois anos com idosos acima de 70 anos discriminou-se o medo de queda como transitório ou persistente. O medo transitório foi definido operacionalmente como um novo relato durante o seguimento de dois anos e que não se mantinha em uma nova entrevista após três meses, e o medo persistente, como tendo uma duração maior que seis meses, sendo captado por duas entrevistas. A incidência acumulada em dois anos foi de 20,6\% para o medo transitório e 30,9\% para o medo persistente. Mesmo não tendo vivenciado recorrência do evento no período estudado, isto não significa necessariamente que se tratou de um evento benigno, já que, em idosos, mesmo eventos transitórios de saúde podem causar incapacidades. Este estudo sugere que ambos os medos possuem fatores de risco compartilhados, o que implica em processos patológicos comuns, mas que em longo prazo ainda não foram investigados quanto às suas consequências. $^{24}$

\section{Fatores associados ao medo de quedas}

Assim como as quedas, o medo de quedas é um fenômeno multidimensional; portanto, ele tem determinantes físicos, psicológicos, sociais e funcionais. Os fatores de risco para o desenvolvimento do medo de quedas, descritos em vários estudos, estão muitas vezes centrados nos aspectos relacionados à capacidade física e fatores sociodemográficos.

Estudos mostram que alterações de marcha e equilíbrio, histórico de quedas, ser do sexo feminino, ter baixo nível de atividade física, usar dispositivos de auxílio à marcha, presença de dor, artrose, deficit visual e ter mais de 80 anos são fatores associados ao medo de quedas. ${ }^{4,18,25}$

Entretanto, na literatura acerca dos construtos envolvidos no medo de quedas, pouco se investiga quanto aos fatores psicológicos e sociais envolvidos. Por ser um fenômeno eminentemente psicológico e subjetivo, é mais difícil de ser avaliado do que os aspectos físicos, mais objetivos. No entanto, a importância de avaliar os aspectos psicossociais neste contexto é evidente, chamando a atenção para a necessidade de estudos que os incluam em seus modelos teóricos. ${ }^{2}$
Na literatura pertinente, está bem estabelecido que o aumento da idade e o sexo feminino são fatores associados a uma maior prevalência do medo de queda. ${ }^{12}$ Em relação ao sexo, a principal hipótese que pode explicar esses achados é aquela relacionada à questão cultural e de gênero, na qual se afirma que os homens têm receio de relatar medo ou preocupação.

\section{Fatores clínicos e funcionais relacionados ao medo de quedas}

Em relação às alterações de equilíbrio e de marcha, estudos mostraram que os indivíduos com medo de cair adaptam sua forma de deambular, forma esta que é descrita como "marcha precavida”. Já que a maior parte das quedas ocorre durante o movimento e a marcha é uma habilidade necessária para realizar diversas tarefas do dia a dia, o equilíbrio dinâmico parece ser importante e estar diretamente relacionado ao medo. ${ }^{26}$ Reelick e colaboradore ${ }^{27}$ encontraram uma velocidade de marcha menor naqueles que apresentaram medo, e os idosos com menor nível de medo usaram mecanismos adaptativos para aperfeiçoar o equilíbrio, muitas vezes utilizando-se de dispositivos de auxílio de marcha.

O estudo realizado por Kirkwood e colaboradore ${ }^{28}$ teve como objetivo estudar os parâmetros da marcha que seriam sensíveis para discriminar idosos com medo de quedas. Neste estudo, observou-se que o comprimento do passo e a velocidade de marcha mostraram associação com o medo, o que evidencia uma maior instabilidade da marcha e, consequentemente, maior risco de quedas.

Recentemente, alguns estudos postularam a associação entre medo e capacidade funcional. ${ }^{17-19}$ A restrição de atividades é um componente comportamental importante do medo. Algumas explicações encontradas relacionaram os idosos que apresentaram histórico de queda com alguma sequela a restrições voluntárias de sua participação em atividades. Essa restrição pode ser ou não temporária. Para alguns indivíduos, ela pode diminuir ao longo do tempo após a recuperação da lesão; para outros, pode se manter por mais tempo. Essa restrição leva ao declínio físico, com consequente inatividade física e isolamento 
social..$^{17-19}$

Em um modelo teórico de fatores associados a quedas, o medo de quedas pode levar a uma restrição excessiva de atividades, o que causaria atrofia muscular, prejuízo no equilíbrio, marcha alterada, comprometimento do condicionamento físico e, por último, novos episódios de quedas. Esse modelo propõe que o impacto negativo do medo de quedas na função física é mediado pela restrição de atividades. Porém, outros construtos devem ser adicionados a esse ciclo, entre eles os fatores psicossociais, como qualidade de vida, depressão e isolamento social. ${ }^{2}$

No estudo de Dias e colaboradores ${ }^{23}$ que investigou características associadas à restrição de atividades por medo de cair, não houve associação com as atividades básicas de vida diária (ABVDs), o que não aconteceu com as atividades instrumentais de vida diária (AIVDs). Isso pode ser explicado pela hierarquia das capacidades funcionais, mostrando que indivíduos com e sem medo possuem desempenho similares nas ABVDs, mas não em atividades mais complexas do dia a dia.

Outros fatores físicos que foram relacionados ao medo de quedas são as alterações visuais, a presença de múltiplas morbidades e o uso de dispositivos de auxílio de marcha..$^{19,20,25}$

\section{Fatores psicossociais relacionados ao medo de quedas}

Alguns poucos estudos têm dado a devida importância aos fatores psicológicos e sociais que estão envolvidos no processo de desenvolvimento do medo de quedas. De acordo com os resultados obtidos por Howland e colaboradores, ${ }^{29}$ a intensidade do medo está associada com o número e a gravidade das quedas vivenciadas, o que é consistente com a teoria do condicionamento clássico. Desta maneira, hipoteticamente, o indivíduo faria uma associação causal entre determinadas atividades e as quedas e lesões que a acompanharam, fazendo com que a repetição de tais atividades gerasse ansiedade.

De forma consistente com essa teoria, a redução das atividades causada pela ansiedade é uma adaptação de curto prazo, mas que em longo prazo contribui para aumentar os pensamentos catastróficos relacionados às quedas.
Porém, mesmo os que não vivenciaram a queda podem ter esses pensamentos, seja pelo testemunho de uma queda, seja pela percepção de seus próprios riscos. ${ }^{2}$

Outro ponto que deve ser considerado é que a percepção do indivíduo sobre seu próprio risco de cair pode alterar seu comportamento. Boyd e Stevens ${ }^{30}$ apontaram que $70 \%$ dos idosos relataram ter conhecimento da necessidade de realizar atividades físicas para prevenir quedas, mas ainda assim não as realizavam, o que sugere que não é necessário apenas o conhecimento, mas sim uma mudança de comportamento, e que o planejamento de programas de prevenção de quedas deve levar em consideração estes aspectos.

Outras causas envolvidas com o medo de queda são: o receio da incapacidade e da perda de independência, o medo da dor e do sofrimento e o receio de que a queda seja um sinal de declínio físico e até de morte. ${ }^{31}$

Poucos trabalhos abordam a relação entre a cognição e o medo. Um estudo encontrou uma associação inversa entre memória e relato de medo de quedas, sugerindo que idosos com declínio cognitivo tendem a subestimar o relato de medo, sendo esta uma das características das mudanças psicológicas dos idosos com declínio de memória. Ou seja, a memória pode ser considerada mais um aspecto que pode influenciar o medo. ${ }^{32}$

Sintomas depressivos e medo foram investigados por Oh-Park e colaboradores,${ }^{24}$ que descreveram que altos escores na Escala de Depressão Geriátrica (EDG) foram fatores de risco para o medo de quedas incidente. Eles apontaram que estudos de neuroimagem têm mostrado que os substratos neurais afetados na depressão são as áreas frontais e límbicas, amígdala e hipocampo, áreas neurais afetadas também no desenvolvimento da ansiedade e do medo.

Hadjistavropoulos e colaboradores ${ }^{2}$ alertam para um novo modelo de contextualização do medo de quedas, e apontam para a necessidade de novos estudos que deem ênfase aos aspectos psicológicos e sociais, como restrição de atividades, ansiedade, apoio social, pensamentos em relação a quedas e autoeficácia.

\section{Conclusões}

Com o crescimento da população idosa, 
torna-se cada vez mais importante identificar idosos com risco de declínio funcional, permitindo a adequação dos serviços de saúde às reais necessidades relacionadas ao processo de envelhecimento. Dentre os fatores que podem causar incapacidades funcionais, está o medo de quedas em idosos, que deve ser considerado e incluído em avaliações geriátricas e gerontológicas, permitindo assim sua identificação precoce e intervenções específicas.

Programas de prevenção de quedas, bem estabelecidos como sendo eficazes e de extrema importância para a manutenção da saúde nos idosos, não necessariamente abordam a questão do medo relacionado às quedas. Por esta ser uma condição influenciada tanto por questões físicas como psicológicas, sociais e emocionais, a abordagem específica para o medo de quedas deve estar inserida nos programas de prevenção de quedas e ser utilizada por uma equipe multidisciplinar.

\section{Referências}

1. Murphy J, Isaacs B. The post-fall syndrome. A study of 36 elderly patients. Gerontology. 1982;28(4):26570 .

2. Hadjistavropoulos T, Delbaere K, Fitzgerald TD. Reconceptualizing the role of fear of falling and balance confidence in fall risk. J Aging Health. 2011;23(1):3-23.

3. Legters K. Fear of falling. Phys Ther. 2002;82(3):264-72.

4. Friedman SM, Munoz B, West SK, Rubin GS, Fried LP. Falls and fear of falling: which comes first? A longitudinal prediction model suggests strategies for primary and secondary prevention. J Am Geriatr Soc. 2002;50(8):1329-35.

5. Tinetti ME, Powell L. Fear of falling and low selfefficacy: a case of dependence in elderly persons. J Gerontol. 1993;48 Spec No:35-8.

6. Lachman ME, Howland J, Tennstedt S, Jette A, Assmann S, Peterson EW. Fear of falling and activity restriction: the survey of activities and fear of falling in the elderly (SAFE). J Gerontol B Psychol Sci Soc Sci. 1998;53(1):P43-50.

7. Lawrence RH, Tennstedt SL, Kasten LE, Shih J, Howland J, Jette AM. Intensity and correlates of fear of falling and hurting oneself in the next year: baseline findings from a Roybal Center fear of falling intervention. J Aging Health. 1998;10(3):267-86.

8. Bandura A, Adams NE, Beyer J. Cognitive processes mediating behavioral change. J Pers Soc Psychol. 1977;35(3):125-39.
9. Bandura A. The assessment and predictive generality of self-percepts of efficacy. J Behav Ther Exp Psychiatry. 1982;13(3):195-9.

10. Bandura A. Self-efficacy: toward a unifying theory of behavioral change. Psychol Rev. 1977;84(2):191-215.

11. Delbaere K, Close JC, Mikolaizak AS, Sachdev PS, Brodaty H, Lord SR. The Falls Efficacy Scale International (FES-l). A comprehensive longitudinal validation study. Age Ageing. 2010;39(2):210-6.

12. Scheffer AC, Schuurmans MJ, van Dijk N, van der Hooft T, de Rooij SE. Fear of falling: measurement strategy, prevalence, risk factors and consequences among older persons. Age Ageing. 2008;37(1):19-24.

13. Tinetti ME, Richman D, Powell L. Falls efficacy as a measure of fear of falling. J Gerontol. 1990;45(6):P239-43.

14. Stretton CM, Latham NK, Carter KN, Lee AC, Anderson CS. Determinants of physical health in frail older people: the importance of self-efficacy. Clin Rehabil. 2006;20(4):357-66.

15. Jorstad EC, Hauer K, Becker C, Lamb SE, ProFANE Group. Measuring the psychological outcomes of falling: a systematic review. J Am Geriatr Soc. 2005;53(3):501-10.

16. Yardley L, Beyer N, Hauer K, Kempen G, Piot-Ziegler C, Todd C. Development and initial validation of the Falls Efficacy Scale-International (FES-l). Age Ageing. 2005;34(6):614-9.

17. Murphy SL, Williams CS, Gill TM. Characteristics associated with fear of falling and activity restriction in community-living older persons. J Am Geriatr Soc. 2002;50(3):516-20.

18. Martin FC, Hart D, Spector T, Doyle DV, Harari D. Fear of falling limiting activity in young-old women is associated with reduced functional mobility rather than psychological factors. Age Ageing. 2005;34(3):281-7.

19. Kempen GI, van Haastregt JC, McKee KJ, Delbaere K, Zijlstra GA. Socio-demographic, health-related and psychosocial correlates of fear of falling and avoidance of activity in community-living older persons who avoid activity due to fear of falling. BMC Public Health. 2009;9:170.

20. Chu CL, Liang CK, Chow PC, Lin YT, Tang KY, Chou MY, et al. Fear of falling (FF): Psychosocial and physical factors among institutionalized older Chinese men in Taiwan. Arch Gerontol Geriatr. 2011;53(2):e232-6.

21. Camargos FFO, Dias RC, Dias JMD, Freire MTF. Adaptação transcultural e avaliação das propriedades psicométricas da Falls Efficacy Scale-International em idosos brasileiros (FES-I-BRASIL). Rev Bras Fisioter. 2010;14(3):237-43.

22. Lopes KT, Costa DF, Santos LF, Castro DP, Bastone AC. Prevalência do medo de cair em uma população de idosos da comunidade e sua correlação com 
mobilidade, equilibrio dinâmico, risco e histórico de quedas. Rev Bras Fisioter. 2009;13(3):223-9.

23. Dias RC, Freire MT, Santos EG, Vieira RA, Dias JM, Perracini MR. Characteristics associated with activity restriction induced by fear of falling in community-dwelling elderly. Rev Bras Fisioter. 2011;15(5):406-13.

24. Oh-Park M, Xue X, Holtzer R, Verghese J. Transient versus persistent fear of falling in communitydwelling older adults: incidence and risk factors. J Am Geriatr Soc. 2011;59(7):1225-31.

25. Kressig RW, Wolf SL, Sattin RW, O’Grady M, Greenspan A, Curns A, et al. Associations of demographic, functional, and behavioral characteristics with activityrelated fear of falling among older adults transitioning to frailty. J Am Geriatr Soc. 2001;49(11):1456-62.

26. Maki BE. Gait changes in older adults: predictors of falls or indicators of fear. J Am Geriatr Soc. 1997;45(3):313-20.

27. Reelick MF, van lersel MB, Kessels RP, Rikkert MG. The influence of fear of falling on gait and balance in

Recebido: 05/09/2013.

Revisado: 26/10/2013.

Aprovado: 06/12/2013. older people. Age Ageing. 2009;38(4):435-40.

28. Kirkwood RN, de Souza Moreira B, Vallone ML, Mingoti SA, Dias RC, Sampaio RF. Step length appears to be a strong discriminant gait parameter for elderly females highly concerned about falls: a cross-sectional observational study. Physiotherapy. 2011;97(2):126-31.

29. Howland J, Lachman ME, Peterson EW, Cote J, Kasten L, Jette A. Covariates of fear of falling and associated activity curtailment. Gerontologist. 1998;38(5):549-55.

30. Boyd R, Stevens JA. Falls and fear of falling: burden, beliefs and behaviours. Age Ageing. 2009;38(4):423-8.

31. Yardley L, Smith H. A prospective study of the relationship between feared consequences of falling and avoidance of activity in community-living older people. Gerontologist. 2002;42(1):17-23.

32. Uemura K, Shimada H, Makizako H, Yoshida D, Doi T, Tsutsumimoto K, et al. A lower prevalence of self-reported fear of falling is associated with memory decline among older adults. Gerontology. 2012;58(5):413-8.

\section{Flávia M. Malini}

Departamento de Epidemiologia. Instituto de Medicina Social. Universidade do Estado do Rio de Janeiro. Rio de Janeiro, RJ, Brasil.

\section{Cláudia S. Lopes}

Departamento de Epidemiologia. Instituto de Medicina Social. Universidade do Estado do Rio de Janeiro. Rio de Janeiro, RJ, Brasil.

\section{Roberto A. Lourenço}

Departamento de Medicina Interna. Faculdade de Ciências Médicas. Universidade do Estado do Rio de Janeiro. Rio de Janeiro, RJ, Brasil. 